\title{
Specialist palliative care improves the quality of life in advanced neurodegenerative disorders: NE-PAL, a pilot randomised controlled study
}

Simone Veronese

Fondazione Assistenza e Ricerca in Oncologia (FARO) Onlus, Turin, Italy

G Gallo

Fondazione Assistenza e Ricerca in Oncologia (FARO) Onlus, Turin, Italy

AValle

Fondazione Assistenza e Ricerca in Oncologia (FARO) Onlus, Turin, Italy

CCugno

Fondazione Assistenza e Ricerca in Oncologia (FARO) Onlus, Turin, Italy

AChiò

Department of Neuroscience,

ALS Centre, University of Turin, Turin, Italy

ACalvo

Department of Neuroscience, ALS Centre, University of Turin, Turin, Italy

P Cavalla

Department of Neuroscience, ALS Centre, University of Turin, Turin, Italy

M Zibetti

Department of Neuroscience, ALS Centre, University of Turin, Turin, Italy

C Rivoiro

Agenzia Regionale Servizi Sanitari Piemonte ARESS Piemonte, Turin, Italy

D J Oliver

Centre for Professional Practice, University of Kent, Chatham, UK

Correspondence to

Dr Simone Veronese

Fondazione Assistenza e Ricerca in Oncologia (FARO) onlus

Via Oddino Morgari 12

10125, Turin

Italy

simone.veronese@fondazionefaro.it 


\section{Abstract}

\section{Background}

This study analysed the impact on palliative care outcomes of a new specialist palliative care service for patients severely affected by amyotrophic lateral sclerosis (ALS/MND), multiple sclerosis, Parkinson's disease and related disorders (multiple system atrophy progressive supranuclear palsy, MSA-PSP).

\section{Methods}

The design followed the Medical Research Council Framework for the evaluation of complex interventions. A phase II randomised controlled trial (RCT) was undertaken comparing an immediate referral to the service (FT, fast track) to a 16-week wait (standard track (ST), standard best practice) using a parallel arm design. The main outcome measures were Quality of Life (measured with Schedule for the Evaluation of Individual Quality of Life Direct Weight, SEIQoL- DW) and burden of the carers (Caregivers Burden Inventory, CBI), with secondary outcomes of symptoms, psychosocial and spiritual issues.

Results

50 patients severely affected by neurodegenerative conditions and their informal family carers were randomized: $25 \mathrm{FT}, 25 \mathrm{ST}$. At baseline (T0) there were no differences between groups. 4 patients died during the follow-up ( 2 FT, 2 ST) and 2 patients dropped out before the end of the study.After 16 weeks (T1), Ft participants scored significant improvement in the SEIQoL-DW index, pain, dyspnea, sleep disturbance and bowel symptoms.

\section{Conclusions}

This exploratory RCT provides evidence that no harm was experienced by SPCS for patients severely affected by neurodegenerative disorders. There was an improvement in quality of life and physical symptoms for neurological patients in palliative care. Caregiver burden was not affected by the service. 


\section{Introduction}

Palliative care aims at improving quality of life $(\mathrm{Q} o \mathrm{~L})$ of patients and their families facing problems associated with incurable, progressive and life-limiting disease by means of the impeccable assessment and treatment of symptoms and other psycho- logical, social and spiritual issues. ${ }^{1}$ Patients affected by progressive neurodegenerative conditions suffer a high burden of symptoms ${ }^{23}$ and issues that are often managed by specialist palliative care teams. ${ }^{4}$ However, there is a challenge in providing specialist palliative care service (SPCS) for individuals with advanced neurological conditions as there is a great variability in disease trajectories and symptom profiles. This has been considered in the discussion of the provision of palliative and end- of-life care for this patient group, ${ }^{4}$ and there is evidence that the suggested triggers for consideration of care may be of help - these being patient request, family request, dysphagia, cognitive decline, dyspnoea, repeated infections (in particular, aspiration pneumonia), weight loss and marked decline in condition. ${ }^{45}$

Although SPCS has been advocated in the care of patients with neurological dis- eases, ${ }^{6}$ there is very little available evidence about the impact of SPCS on the typical palliative care outcomes. Moreover, there is little definitive evidence for the effectiveness of palliative care generally. One review has shown that the evidence for benefit from specialised palliative care is sparse and limited by methodological shortcomings. ${ }^{7}$ Carefully planned trials, using a standardized palliative care intervention and measures constructed specifically for this population, are needed. One project-ENABLE $2^{8}$-did show in an randomised controlled trial (RCT) that QoL and depression were improved by the SPCS in patients with advanced cancer, even though the physical symptoms were not. A literature review suggested that SPCSs provide significant benefits on patients' pain and other symptoms for patients with advanced cancer. $^{9}$

Within the care of people with neurological conditions, there has been little research, although one study, an RCT using a delayed intervention model, did show that early specialist palliative care did help people with multiple sclerosis (MS). There were positive results for the management of nausea and sleeping problems to some degree, with the effect strongest after initial contact with the clinical service. There was also a positive impact on informal carer wellbeing and user satisfaction with the service was high among patients with MS, carers and especially other healthcare professionals. ${ }^{10}{ }^{11}$ No studies were found in the literature exploring the impact of an SPCS for people severely affected by amyotrophic lateral sclerosis/motor neurone disease (ALS/MND), MS or Parkinson's disease (PD) when considered as a whole group of people with progressive neurological patients (refs. 12 and 13, p.220).

The aim of this project is to investigate if the involvement of SPCS would affect individual QoL of patients severely affected by neurodegenerative conditions and the burden of care of their informal family carers.

\section{Methods}

The study adopted a phase 2 pilot $\mathrm{RCT}^{14}$ using the fast track (FT) versus standard track (ST) design, also called the waiting list study, adapted from a previous study on MS. ${ }^{15}$ This allowed comparison of an SPCS and standard care. This paper has been reported in line with the CONSORT guidelines. 


\section{Participants}

Participants were adults severely affected by ALS/ MND, MS or PD. Informal family carers of the patients were also enrolled if they wished. Patients were resident in Turin city or in the metropolitan area as this was the area where the SPCS-Fondazione Assistenza e Ricerca Oncologia (FARO)-is able to provide care. The severity of the disease was defined according to the Gold Standards Framework prognostic indicators specific for the different conditions ${ }^{16}$ as shown in table 1-ALS/MND severe disease, MS Expanded Disability Status Scale ${ }^{17}$ equal to or greater than 8.5, PD Hoehn and $Y_{a h r^{18}} \geq 4$. Participants were excluded if the inclusion criteria were not satisfied, if the cognitive state was compromised so that they could not complete the outcome measures (augmentative or alternative communication aids were incentivised where possible) or if consent to participation was refused. If the informal family carer was not available or the patient did not wish their participation, only the patient was enrolled.

Participants were referred to the study by the medical specialists working in the tertiary clinics for the specific neurological disease groups of two main hospitals of Turin. As this was a pilot RCT, no sample size based on the statistical calculation was calculated a priori, and a sample of 50 participants, allocated 25/group, was chosen as it was estimated that this increase in the number of patients could be coped with by the existing palliative care service.

Every week, two patients with the same disease and similar clinical features (eg, both with ALS/MND and receiving non-invasive ventilation) were assessed at baseline (TO), and then randomised one to the FT group and the other to the ST group using a parallel arm design. The randomisation was undertaken by placing two unrecognisable white folders, containing the two patients' charts, on a secretary's desk. The secretary was asked to pick up one randomly and this was chosen as the FT patient, the other patient went into the ST group. Patients allocated in the FT group received the SPCS immediately, whereas those allocated in the ST group waited 16 weeks (T1) before receiving the palliative care input-figure 1 (loaded as a separate file). After T1 all the participants could receive the SPCS, if they wished, for as long as necessary. Participants randomised to the ST group had a waiting list of 16 weeks after allocation, and during this period they received the standard care, provided by primary medicine and hospital specialists. Patients were asked to identify their main informal caregiver and to decide whether they could be involved and complete the tools for caregivers.

The SPCS was provided by FARO Foundation, ${ }^{19}$ which provided a team, trained and expert in palliative care, comprising a physician, a nurse, a psychologist and a physiotherapist. The team members visit the patients and the family on a regular basis depending on the palliative care needs and issues that are assessed in that assistance. On average, patients are seen at least weekly by a team member and all patients are discussed at a team meeting every 2 weeks. Volunteers are also available if social support is required. The team can assess symptoms, prescribe medications, provide nursing care and physical therapies as well as psychological support and bereavement care. Team members and study personnel could not be blind to participants, as they were involved in their care.

\section{Measures}

The outcome measures were applied at T0 (before randomisation) and at T1 (when only the FT group had received the SPCS care).The main outcomes were:

- Patients' individual QoL measured with the Schedule for the Evaluation of Individual 
Quality of Life Direct

Weight (SEIQoL-DW), ${ }^{20}$ a validated tool to measure this domain in these patients' group; ${ }^{21-23}$

- Family informal carers' burden of care measured with

the Caregiver Burden Inventory. ${ }^{24}$

The secondary outcome measures were physical, psychological, social and spiritual issues that had particular relevance to this patient group in an earlier qualitative study. ${ }^{3} \mathrm{~A}$ full list of the outcomes and the relative measurement tools is shown in table 2.

Analysis

Analysis was undertaken from the outcome measures at baseline (T0), after 16 weeks (T1) and the difference between the mean scores (T1-TO). In order to appraise the potential benefit of the SPCS, clinical and statistical significance were considered. The SPSS 15 software package was used for data analysis.

Clinical significance provides information on whether a treatment is effective enough to change a patient's diagnostic label or to make a significant change in a patient's condition. For pain and sleep disturbance, this change has been described as greater than $13 \%$ and $10 \%$, respectively. ${ }^{28}$

${ }^{29}$ It has been suggested that a change of $50 \%$ of the SD of any QoL tool can be considered a clinically significant change. This translates to a change of 8-10 points on a 100-points scale. ${ }^{30}$

The clinical interpretation of the results in this study has been categorised in three possible groups:

- No clinical significance if the difference between T1 and T0 is lower than $10 \%$ of the scale used to measure that domain;

- Moderate clinical significance if that difference is between $10-19 \%$ of the same scale;

- Relevant clinical significance when the difference is $20 \%$ or higher.

The statistical analysis included:

- Descriptive statistics to evaluate the comparability of the two study groups

- Group comparison test to detect differences between the two study groups that can be caused by the intervention.

- The aim of this procedure is to determine the efficacy analysis. Depending on the characteristics of the vari- ables, the analysis of covariance (ANCOVA), independ- ent $t$ test or $\chi^{2}$ test were used to highlight statistical changes between the two groups.

- The null hypothesis of this study is that no changes are tobe found between the FTand ST groups after the interven- tion represented by the provision of the SPCS in the treated group versus the best standard care in the control group.

- The $\alpha$ value, determining the statistical significance foundin this study, is set at 0.05 . Bonferroni adjustments were applied for the ANCOVA test of the variables which were significantly different between the groups at $\mathrm{T} 1$.

Ethics

As there was no existing service for people with progressive neurological disease, it was felt to be ethical to delay the service for 16 weeks for the control group, who were offered care at the end of this period. Patients in the control group who experienced symptoms at baseline were advised to consult their general practitioner or neurologist for advice. 
Ethical approval was obtained from the Ethics Committee of the two hospitals-Department of Neuroscience, University of Torino and S. Luigi Gonzaga Hospital, Orbassano-and the University of Kent Ethics Committee, University of Kent at Canterbury, UK, as this study was part of a Doctoral study there.

\section{Results}

Demographics, recruitment, attrition, mortality

From March 2008 to February 2009, 52 potential participants were referred to the study. Two participants refused to participate, both with MS: one did not feel seriously ill and for this reason did not want to be cared for by an SPCS; the other had recently been diagnosed with breast cancer and was going to start chemotherapy. Of the 50 patients enrolled, $30(60 \%)$ were male and 20 (40\%) female. The mean age was 61 years and $8(16 \%)$ were aged under 44 years, 17 (34\%) 45-65 years and 25 (50\%) over 65 years. The diagnosis was ALS/MND for 16 (32\%), MS for $18(36 \%)$ and PD for $16(32 \%)$.

Five patients did not wish their carer to be involved, and so 45 family carers were included in the study -24 wives, 12 husbands, 4 daughters, 1 son, 1 mother and 1 sister.

Two patients were not able to complete the SeiQol-DW interview, and therefore their QoL index at baseline is missing. All patients were able to complete the assessment of symptoms. All participants but two completed the psychosocial and spiritual assessment.

At baseline, no differences were found in the two groups for demographics, clinical features, disability level (see online supplementary appendix table S1) and the outcome measure (see online supplementary appendix table S2), showing that the randomisation was successful.

At T1, after FT had received the SPCS, the mortality in the two groups was the same: 2 patients died in the FT group and 2 in the ST group, during the 16 weeks. Two patients dropped out, both in the FT group: a patient with ALS/MND and a patient with MS, both of whom decided not to continue the study for personal reasons after the baseline assessment.

Results of intervention

Outcome measures at T1 revealed a significant improvement $(p<0.05)$ for the FT group in one main outcome, the individual QoL of patients, and in five secondary outcomes, all in the physical symptoms: pain, breathlessness, sleep disturbance, intestinal and urinary symptoms and mouth discomfort-(see online supplementary appendix table S3).

Clinical significance: In order to compare the results between the two groups that could be caused by the intervention of the SPCS, new variables were created by subtracting the mean results of the test at T0 (baseline) from the ones obtained at T1 (after intervention). These new variables were called 'difference variables' and results are shown in table 3 . The difference reported in percentage was used to assess the clinical significance of the results. These results showed a clinical (>20\%) improvement of the QoL, pain control, breathlessness and sleep disturbance of the patients who received the SPCS. 
Statistical significance: A one-way ANCOVA between-groups analysis was conducted for those variables that showed a statistically significant difference between the two groups after the intervention (T1). The aim of this test was to determine whether by adjusting these results for the covariate represented by the baseline assessment (TO) of the same domains the statistical significance was maintained. The seven variables with these characteristics were: QoL and the physical symptoms (pain control, breathlessness, sleep disorders, urinary symptoms, intestinal symptoms and oral symptoms). The independent variable was the type of intervention: FT/ST. The dependent variable was the score on the SEIQoL-DW and the Numerical Rating Scale scores for the remaining six symptoms at T1. Participants' scores at T0 (baseline) were used as the covariate in this analysis.

A Bonferroni adjustment was applied to the $\alpha$ value that was considered significant at 0.007 .

As shown in table 4, a statistically significant advantage for FT was shown for the QoL, pain, breathless- ness, sleep disturbance and bowel symptoms. Figure 2 (loaded as a separate file) provides an overall picture of the results.

\section{Discussion}

This study aimed to understand the impact of an SPCS on palliative care outcomes of people severely affected by neurodegenerative disorders. The main outcomes were the individual QoL of patients and the burden of care of their informal family carer. The results show that there was a positive improve- ment in QoL for those patients who received the SPCS. The overall QoL was similar to that shown in previous studies as in this study the mean SEIQoL-DW was 62 , similar to $72,{ }^{22} 73^{31}$ for ALS/ MND and 61 for $\mathrm{MS}^{21}$ and 60 for a general palliative care population, ${ }^{32}$ although these results are from a single observation and not over time. In this RCT, the sample of patients who received the SPCS scored over $20 \%$ higher on the SEIQoL index than the control group, and this difference was confirmed statistically.

The other main outcome on the burden of care for families and carers was not found to be affected by the provision of the SPCS. This may reflect the long disease progression and that families had experienced the burden of caring for the patient for many months or years before the SPCS was provided. Moreover, as the SPCS became involved in these later stages, families were confronted with discussion of death and dying, as the patients were so ill. This may have been difficult for them, so that may have felt that the SPCS added to their psychological burdens.

However, symptoms were shown to be significantly improved, both clinically and statistically, for pain, breathlessness, sleep disturbance and bowel symptoms. It is likely that these improvements had a positive impact on the QoL, and therefore can explain the positive impact of the service on patients' QoL. The study on patients with $\mathrm{MS}^{11}$ showed a significant change only in the management of nausea, although there is much anecdotal evidence that SPCS does improve symptom management. ${ }^{33}$

There were positive trends towards improvement of other symptoms and issues, in particular social isolation of the patients, all the other physical symptoms, service satisfaction for both patients and informal family carers, and a help to find a meaning in the experience of the disease. Only for the psychological outcomes was the trend towards a worsening in the treated group, although this was not clinically or statistically significant. It is possible that the impact of professionals so used to death and dying caused difficulties in coping with patients who often think them- selves as chronically ill, rather than terminally ill. Other studies have suggested 
that people with ALS/ MND and other chronic, progressive illnesses may become demoralised and less able to accept changes and work on the more complex and difficult psycho- logical issues. $^{34}$

The study also showed that recruitment was possible for an RCT of this nature as only two potential participants refused to be enrolled and attrition was low with only two patients abandoning the study. The mortality was equal in the two arms. This was shown in the previous study on MS and shows that palliative care does not seem to hasten death. ${ }^{15}$ Thus, it would appear that the methodology of the waiting list procedure is feasible and reliable and can be used to assess palliative care outcomes in palliative care settings for patients with an expected life span of months/years. Low attrition and relatively low missing data confirmed a good impact of the study on the participants who adhered to this study and remained in the protocol for the follow-up with a very low attrition rate.

There are limitations in this study as the population was heterogeneous and formed by patients affected by different clinical conditions and with different trajectories. Some tools were not validated for secondary outcomes, but were chosen to measure specific issues that emerged in a previous needs assessment. ${ }^{35}$ Only one evaluation and no crossover could be carried out over time, so we do not know if the improvement in the measured domains is maintained. Within the practicality of this study, these issues could not be addressed but ideally should have been considered.

\section{Conclusion}

This study represents one of the first published experiences of assessment and evaluation of the impact of an SPCS in non-cancer conditions. Moreover, there are very limited assessments of any palliative care in any patients group, although there has been evidence of early palliative care leading to improved QoL and prognosis in lung cancer. ${ }^{36}$ The lack of evidence of effect on informal carers' well-being should be studied in depth. The approach to relatives who have been providing care to their beloved for a very long time could be different from that successfully adopted to support informal carers of patients with cancer.

Competing interests

None declared.

Ethics approval

Ethical approval was obtained from the Ethics Committee of the two hospitals-Department of Neuroscience, University of Torino and S. Luigi Gonzaga Hospital, Orbassano -and the University of Kent Ethics Committee, University of Kent at Canterbury, UK, as this study was part of a Doctoral study there. 


\section{References}

1 WHO. Definition of palliative care. Secondary definition of palliative care, 2002. http://www.who.int/cancer/palliative/ definition/en/

2 Saleem T, Leigh PN, Higginson IJ. Symptom prevalence among people affected by advanced and progressive neurological conditions-a systematic review. J Palliat Care 2007;23:291-9.

3 Veronese S. Qualitative needs assessment. In: Veronese S, Oliver D, eds. Palliative care for people with neurodegenerative conditions. Saarbrucken: LAP Lambert Academic Publishing, 2013:65-275.

4 NCPC TNCFPC. End of life care in long term neurological conditions: a framework for implementation. National end of life programme, 2010.

5 Shimizu T, Hayashi $\mathrm{H}$, Kato $\mathrm{S}$, et al. Circulatory collapse and sudden death in respiratordependent amyotrophic lateral sclerosis. J Neurol Sci 1994;124:45-55. DH-UK. The National Service Framework for long term conditions. UK: Department of Health, 2005.

7 Zimmermann C, Riechelmann R, Krzyzanowska M, et al. Effectiveness of specialized palliative care: a systematic review. JAMA 2008;299:1698-709. Bakitas M, Lyons KD, Hegel MT, et al. Effects of a palliative care intervention on clinical outcomes in patients with advanced cancer: the Project ENABLE II randomized controlled trial. JAMA 2009;302:741-9.

9 Higginson IJ, Finlay IG, Goodwin DM, et al. Is there evidence that palliative care teams alter end-of-life experiences of patients and their caregivers? J Pain Symptom Manage 2003;25:150-68. Edmonds P, Higginson I, Silber E, et al. Palliative care for people with MS: Final Report. MS Society, 2006.

11 Higginson IJ, McCrone P, Hart SR, et al. Is short-term palliative care cost-effective in multiple sclerosis? A randomized phase II trial. J Pain Symptom Manage 2009; 38:816-26. Higginson I. Health care needs assessment: palliative and terminal care. In: Stevens A, Raftery J, eds. Health care needs assessment. 2nd Series. Oxford: Radcliffe Medical Press, 1997:183-260. Doyle D, Biswas B, Dunlop R, et al. Outcomes measures in palliative care. National Council for Hospice and Specialist Palliative Care Services, 1995. Campbell M, Fitzpatrick R, Haines A, et al. Framework for design and evaluation of complex interventions to improve health. BMJ 2000;321:694-6. Higginson IJ, Hart S, Burman R, et al. Randomised controlled trial of a new palliative care service: compliance, recruitment and completeness of follow-up. BMC Palliat Care 2008;7:7. The-Gold-Standards-Framework. Prognostic Indicator Paper vs 2.25 Secondary Prognostic Indicator Paper vs 2.25 September 2008. http://www.goldstandardsframework.nhs.uk

17 Kurtzke JF. Rating neurologic impairment in multiple sclerosis: an expanded disability status scale (EDSS). Neurology 1983; 33:1444-52. Hoehn MM, Yahr MD. Parkinsonism: onset, progression and mortality. Neurology 1967;17:427-42. 
FARO. Fondazione FARO onlus. Secondary Fondazione FARO onlus 1983.

http://www.fondazionefaro.it/

Hickey AM, Bury G, O'Boyle CA, et al. A new short form individual quality of life measure (SEIQoL-DW): application in a cohort of individuals with HIV/AIDS. BMJ 1996;313:29-33.

Lintern TC, Beaumont JG, Kenealy PM, et al. Quality of Life (QoL) in severely disabled multiple sclerosis patients: comparison of three QoL measures using multidimensional scaling. Qual Life Res 2001;10:371-8. Neudert C, Wasner M, Borasio GD. Patients' assessment of quality of life instruments: a randomised study of SIP, SF-36 and SEIQOL-DW in patients with amyotrophic lateral sclerosis. J Neurol Sci 2001;191:103-9. Lee MA, Walker RW, Hildreth AJ, et al. Individualized assessment of quality of life in idiopathic Parkinson's disease. Mov Disord 2006;21:1929-34. Novak M, Guest C. Application of a multidimensional caregiver burden inventory. Gerontologist 1989;29:798-803. Zigmond AS, Snaith RP. The hospital anxiety and depression scale. Acta Psychiatr Scand 1983;67:361-70. Kempen GI, Suurmeijer TP. The development of a hierarchical polychotomous ADL-IADL scale for noninstitutionalized elders. Gerontologist 1990;30:497-502.

27 Hodkinson HM. Evaluation of a mental test score for assessment of mental impairment in the elderly. Age Ageing 1972;1:233-8.

28 Gallagher EJ, Liebman M, Bijur PE. Prospective validation of clinically important changes in pain severity measured on a visual analog scale. Ann Emerg Med 2001;38: 633-8. Nava Z, Tali N. Determination of the minimal clinically significant difference on a patient visual analog sleep quality scale. J Sleep Res 2003;12:291-8. Sloan J. Asking the obvious questions regarding patient burden. J Clin Oncol 2002;20:4-6. weaknesses of the before-after study design and strategies to improve it. Palliat Med 2009;23:23-8. Temel JS, Greer JA, Muzikansky A, et al. Early palliative care for patients with metastatic non-small-cell lung cancer. N Engl J Med 2010;363:733-42. 
Table 1 - Clinical requirements indicating advanced disease

General indicators of deterioration in neurological diseases

- Progressive deterioration in physical or cognitive function despite optimal therapy

- Complex symptoms

- Swallowing problems leading to respiratory complications

- Speech problems: dysarthria and progressive dysphasia

Specific clinical indicators for the disease groups

ALS/MND

- Evidence of disturbed sleep related to respiratory muscle weakness in

addition to signs of dyspnoea at rest

- Increased cognitive difficulties/barely intelligible speech

- Difficulty swallowing poor nutritional status/weight loss

- Needing assistance with ADLs

- Medical complications, eg, pneumonia, sepsis

- A low vital capacity (below $50 \%$ of predicted, hypercapnia)

Multiple sclerosis

1. EDSS $\geq 8,5$

2. Presence of at least one of the following conditions:

- Significant complex symptoms and medical complications

- Dysphagia (swallowing difficulties) admissions with sepsis and poor nutritional status

- Communication difficulties, eg, dysarthria+fatigue

- Cognitive impairment

- Breathlessness

Movement disorders

1. Hoehn and Yahr stage $\geq 4$

2. No indication for neurosurgical procedures

3. The presence of 2 or more of the following criteria:

- Drug treatment is less effective or an increasingly complex regime of drug treatments

- Reduced independence, need for help with daily living

- Recognition that the condition has become less controlled and less predictable with "off" periods

- Dyskinesias, mobility problems and falls

- Swallowing problems

- Psychiatric signs (depression, anxiety, hallucinations, psychosis)

Adapted from the Gold Standards Framework prognostic indicator guidance.

$A D L$, activity of daily living; ALS/MND, amyotrophic lateral sclerosis motor neurone disease. 
Table 2 Selected domains and outcome measures

Outcomes and general areas

Measurement tool

Primary outcome $1 \quad$ Patients' individual QoL

Primary outcome 2 Caregivers' burden of care

Physical symptoms

Psychological issues

Social issues

Spiritual issues

NRS 0-10
- Pain

- Shortness of breath

- Quality of sleep

- Urinary problems

- Intestinal problems

- Oral symptoms

- Anxiety

- Depression

- Feeling abandoned

- Coping with the disease

- Social isolation

- Service satisfaction

- Meaning of the experience

- Help from faith
Specific domain

The SEIQoL-DW

CBI

NSR 0-10

HADS $^{25}$

NSR 0-10

NSR 0-10

NSR 0-10

NRS 0-10Disability (only at baseline) ADL

- ADL

- IADL tests ${ }^{26}$

Short form of the Mini-mental state test called AMTS ${ }^{2}$

Specific disability scales for the three diagnostic groups

- ALSFRS-R for patients with ALS/MND

- EDSS for patients with $\mathrm{MS}^{17}$

- H\&Y for patients with $\mathrm{PD}^{18}$

$A D L$, activity of daily living; ALS/MND, amyotrophic lateral sclerosis motor neurone disease; ALSFRS-R, Amyotrophic Lateral Sclerosis Functional Rating Scale-Revised; CBI, Caregiver Burden Inventory; AMTS, Abbreviated Mental Test Score; EDSS, Expanded Disability Status Scale; HADS, Hospital Anxiety and Depression Scale; H\&Y, Hoehn and Yahr; IADL, instrumental activity of daily living; MS, multiple sclerosis; NRS, Numerical Rating Scales; PD, Parkinson's disease; SEIQoL-DW, Schedule for the Evaluation of Individual Quality of Life Direct Weight. 
Table 3 Comparison between $\Delta$ means: clinical and statistical significance

\begin{tabular}{|c|c|c|}
\hline Domain & $\begin{array}{l}\text { Differences } \\
\Delta \text { means T1-T0 (SD) } \\
\text { in FT and ST }\end{array}$ & $\begin{array}{l}\text { Clinical significance } \Delta \text { means } \\
\text { T1-T0; FT vs ST }(\Delta \%) \\
\text { Statistical significance }\left(p<0.05^{*}\right)\end{array}$ \\
\hline $\begin{array}{l}\text { Quality of life-SEIQoL-DW index (0-100) } \\
\text { Higher scores=better QoL }\end{array}$ & $\begin{array}{l}\mathrm{FT}=+12.8(12.8) \\
\mathrm{ST}=-7.4(19.3)\end{array}$ & $20.19(20.2 \%)^{*}$ \\
\hline $\begin{array}{l}\text { Pain }(0-10) \\
\text { Lower score=lower symptom }\end{array}$ & $\begin{array}{l}\mathrm{FT}=-0.76(3.2) \\
\mathrm{ST}=+1.65(3.4)\end{array}$ & $-2.41(24.1 \%)^{*}$ \\
\hline $\begin{array}{l}\text { Breathlessness }(0-10) \\
\text { Lower score=lower symptom }\end{array}$ & $\begin{array}{l}\mathrm{FT}=-1.57(2.9) \\
\mathrm{ST}=+0.69(3.1)\end{array}$ & $-2.26(22.6 \%)^{*}$ \\
\hline $\begin{array}{l}\text { Sleep disturbance }(0-10) \\
\text { Lower score=lower symptom }\end{array}$ & $\begin{array}{l}\mathrm{FT}=-1.61(3.2) \\
\mathrm{ST}=+0.52(3.2)\end{array}$ & $-2.14(21.4 \%)^{*}$ \\
\hline $\begin{array}{l}\text { Urinary symptoms }(0-10) \\
\text { Lower score=lower symptom }\end{array}$ & $\begin{array}{l}\mathrm{FT}=-2.09(2.8) \\
\mathrm{ST}=-0.35(4.4)\end{array}$ & $-1.74(17.4 \%)$ \\
\hline $\begin{array}{l}\text { Bowel symptoms }(0-10) \\
\text { Lower score=lower symptom }\end{array}$ & $\begin{array}{l}\mathrm{FT}=-1.90(4.1) \\
\mathrm{ST}=+0.17(3.3)\end{array}$ & $-2.07(20.7 \%)$ \\
\hline $\begin{array}{l}\text { Oral symptoms }(0-10) \\
\text { Lower score=lower symptom }\end{array}$ & $\begin{array}{l}\mathrm{FT}=-0.81(3.1) \\
\mathrm{ST}=+0.43(3.3)\end{array}$ & $-1.24(12.4 \%)$ \\
\hline $\begin{array}{l}\text { Feeling abandoned }(0-10) \\
\text { Higher scores=lower distress }\end{array}$ & $\begin{array}{l}\mathrm{FT}=+0.45(3.7) \\
\mathrm{ST}=-0.22(3.9)\end{array}$ & $0.67(6.7 \%)$ \\
\hline $\begin{array}{l}\text { Coping with the disease }(0-10) \\
\text { Higher scores=better coping }\end{array}$ & $\begin{array}{l}\mathrm{FT}=+0.30(3.6) \\
\mathrm{ST}=+1.22(2.8)\end{array}$ & $-0.92(9.2 \%)$ \\
\hline $\begin{array}{l}\text { Meaning }(0-10) \\
\text { Higher scores=higher meaning }\end{array}$ & $\begin{array}{l}\mathrm{FT}=+0.50(3.6) \\
\mathrm{ST}=-0.95(3.9)\end{array}$ & $1.45(14.5 \%)$ \\
\hline $\begin{array}{l}\text { Help from faith }(0-10) \\
\text { Higher scores=better support }\end{array}$ & $\begin{array}{l}\mathrm{FT}=0.0(1.8) \\
\mathrm{ST}=-0.31(2.6)\end{array}$ & $0.31(3.1 \%)$ \\
\hline $\begin{array}{l}\text { Sense of social isolation (patient } 0-10 \text { ) } \\
\text { Higher scores=lower sense of isolation }\end{array}$ & $\begin{array}{l}\mathrm{FT}=+1.35(3.7) \\
\mathrm{ST}=-1.04(4.7)\end{array}$ & 2.39 (23.9\%) \\
\hline $\begin{array}{l}\text { Service satisfaction (patient } 0-10 \text { ) } \\
\text { Higher scores=higher satisfaction }\end{array}$ & $\begin{array}{l}\mathrm{FT}=+0.85(3.8) \\
\mathrm{ST}=-0.52(2.1)\end{array}$ & $1.37(13.7 \%)$ \\
\hline $\begin{array}{l}\text { Sense of social isolation (carers } 0-10 \text { ) } \\
\text { Higher scores=lower sense of isolation }\end{array}$ & $\begin{array}{l}\mathrm{FT}=+0.50(4.4) \\
\mathrm{ST}=-0.28(4.7)\end{array}$ & $0.78(7.8 \%)$ \\
\hline $\begin{array}{l}\text { Service satisfaction (carers } 0-10 \text { ) } \\
\text { Higher scores=higher satisfaction }\end{array}$ & $\begin{array}{l}\mathrm{FT}=+1.25(3.8) \\
\mathrm{ST}=-0.09(2.6)\end{array}$ & $1.34(13.4 \%)$ \\
\hline $\begin{array}{l}\text { Caregiver burden of care CBI index }(0-96) \\
\text { Lower score=lower burden of care }\end{array}$ & $\begin{array}{l}\mathrm{FT}=-4.20(10.0) \\
\mathrm{ST}=+0.73(9.37)\end{array}$ & $-4.93(5.1 \%)$ \\
\hline
\end{tabular}

- $\Delta=$ is the numerical difference in the mean score T1-T0 between the FT and ST groups.

- $\Delta \%=$ is the difference in the percentage of the mean score T1-TO between the FT and ST groups.

- $\quad \geq 20 \%$ is considered as a relevant significant difference.

- $10-19 \%$ is considered as a moderate clinical significance.

- $\quad<10 \%$ no clinical difference.

*Statistical significance: Significant at $p<0.05$ level.

CBI, Caregivers Burden Inventory; FT, fast track; SEIQoL-DW, Schedule for the Evaluation of Individual Quality of Life Direct Weight; ST, standard track. 
Table 4 Analysis of covariance (ANCOVA) results

Domain ANCOVA test Significant $p<0.007$ Partial $\eta^{2}$

Quality of life $\quad(F 1,38)=22,57 \quad 0.000 \quad 0.373$

Pain $\quad(F 1,41)=19, \quad 0.000 \quad 0.320$

29

Breathlessness $(\mathrm{F} 1,41)=15, \quad 0.000 \quad 0.278$

$\begin{array}{llll} & 78 \\ \text { Sleep } & (\mathrm{F} 1,41)=11, & 0.002 & 0.219\end{array}$

$\begin{array}{llll}\text { disturbances } & 47 & & \\ \text { Bowel } & (F 1,41)=8,2 & 0.006 & 0.168\end{array}$

symptoms 6

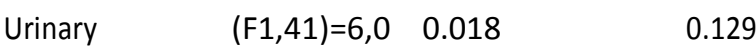

symptoms 8

$\begin{array}{llll}\text { Mouth } & (\mathrm{F} 1,41)=4,3 & 0.042 & 0.097\end{array}$

Significant $p$ value at $<0.007$ (Bonferroni adjustment). F1=ANCOVA test result. 
Figure 1 Flow chart of the study loaded as a separate file (GP, general practitioner; SPCS, specialist palliative care service; ST, standard track).
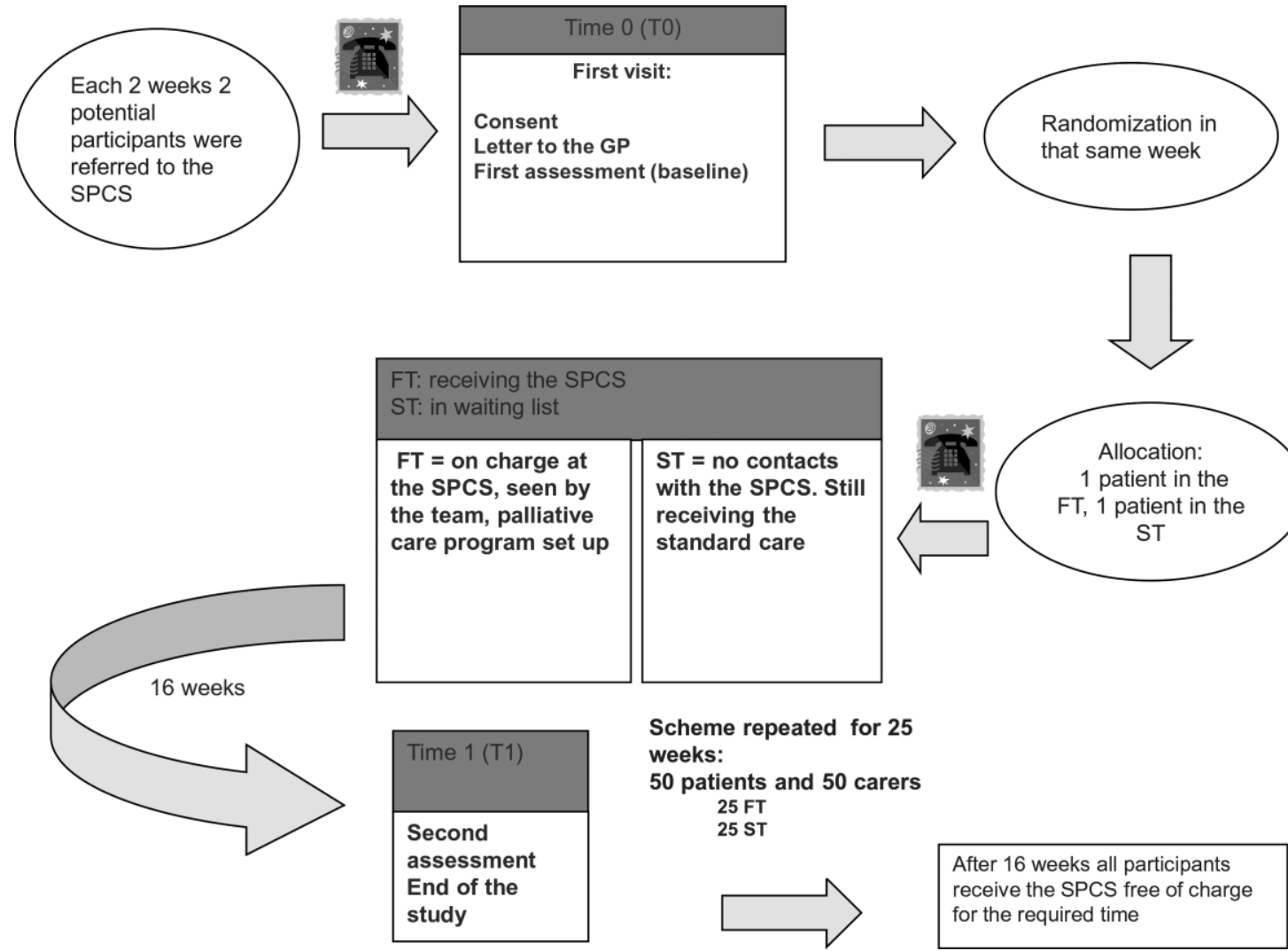
Figure 2 Overall results loaded as a separate file (ANCOVA, analysis of covariance; FT, fast track; QoL, quality of life ST, standard track.

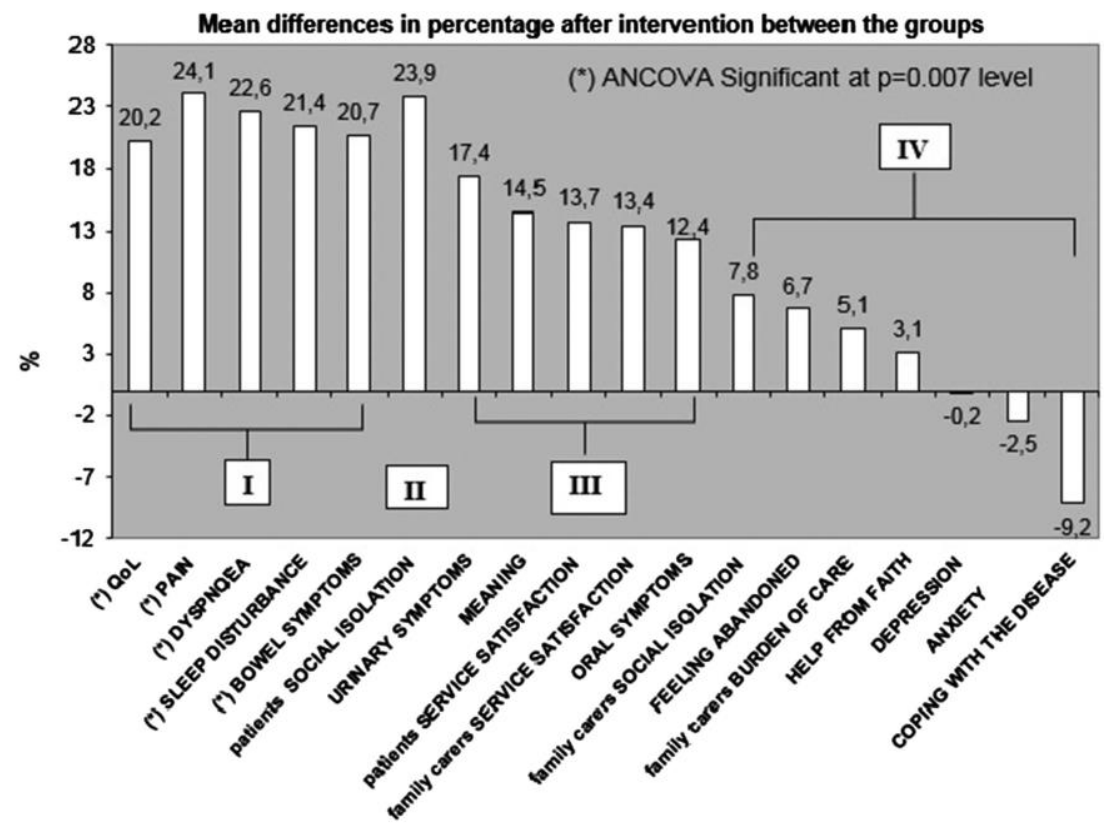

\section{Comparison between $\Delta$ means T1-T0 (in \%) between the FT and ST groups.}

- I bars represent domains with both a statistical $(p<0.007)$ and clinically relevant $(\Delta>20 \%)$ advantage for FT versus ST

- II bar represents a clinical relevant advantage of FT vs ST, but no statistically significant difference.

- III bars represent clinical moderate advantage ( $\Delta 10-19 \%)$ for FT vs ST, but no statistically significant difference.

- IV bars represent domains where no statistical nor clinical significant differences were found between the groups.

(*) $\mathrm{p}<0.007$ 
